\title{
Analytical and Functional Aspects of Antibody Sialylation
}

\author{
Johannes Stadlmann • Martin Pabst • \\ Friedrich Altmann
}

Published online: 14 April 2010

(C) The Author(s) 2010. This article is published with open access at Springerlink.com

\begin{abstract}
Materials and methods This review focuses on the role of antibody sialylation and methods for its quantitation. The recent attribution of the anti-inflammatory activity of IgG to the sialylation of its glycans in the Fc region has raised interest in the fine structure and analysis of the glycans. The antiinflammatory fraction of intravenous $\operatorname{IgG}$ could be isolated with the Sambucus nigra lectin. Experimental strategies for the assessment of antibody sialylation are discussed.

Results Thorough analysis of the lectin-binding fraction revealed that the antibody Fc region only binds to $S$. nigra lectin when two sialic acids are present, whereas for other glycoprotein ligands, one sialic acid appears sufficient.
\end{abstract}

Keywords Antibody glycosylation · sialic acid · IVIG · anti-inflammatory

\section{Introduction}

Antibody glycosylation constitutes the molecular foundation of a number of highly interesting features, including increased cytotoxic toxicity of nonfucosylated $\mathrm{IgG}$ or diminished efficacy conferred by sialylation, as has been discussed in recent reviews [1, 2]. The groundbreaking articles by Anthony et al. [3] and Kaneko et al. [4], on the other hand, established Fc sialylation as a key feature for the anti-inflammatory activity of intravenous IgG (IVIG).

J. Stadlmann $\cdot$ M. Pabst $\cdot$ F. Altmann $(\bowtie)$

Department of Chemistry, University of Natural

Resources and Applied Life Sciences (BOKU),

Muthgasse 18,

1190 Vienna, Austria

e-mail: friedrich.altmann@boku.ac.at
Emphasizing the immunology, these articles, however, did not adequately define the meaning of "sialylation". The same group also suggested that sialylation of antibodies may be adapted to the needs of the immune system, as sialylation of serum-derived IgG was greatly diminished after active immunization of mice [4]. In a more recent study, increased sialylation and galactosylation were found on purified, antigen-specific IgG (alloantibodies) from pregnant mothers [5]. Along with a concomitant decrease of fucosylation, this also presents an adaptation of $\mathrm{IgG}$ glycosylation to respective homeostatic requirements but in the opposite direction. Obviously, such studies depend on reliable quantitative analysis of neutral and sialylated antibody $\mathrm{N}$-glycans. In this review, we discuss promising experimental approaches aimed at the unbiased analysis of all IgG glycoforms and present recent results, where quantitative data on antibody glycosylation have brought unexpected insight into the structural basis of a biological function of glycans. A more comprehensive discussion on glycosylation analysis in general can be found in reviews with a broader scope, such as that of Huhn et al. [6].

\section{Strategies for IgG Glycosylation Analysis}

The bewildering variety of approaches for glycosylation analysis falls into two distinct categories. One strategy works with released glycans. The second strategy analyzes glycopeptides, where the Fc glycosylation can be analyzed site specifically without interference by Fab glycans or other contaminating glycoproteins [7, 8]. Glycopeptides from the Fab region can, however, only be analyzed in the case of monoclonal antibodies (mAbs). With polyclonal IgG, the glycopeptide-based approaches inherently ignore part of the whole picture. 
In the "glycoproteomic" approach, IgG is reduced and, optionally, $S$-alkylated, digested with trypsin, and the peptides are subjected to LC-ESI-MS [7-11]. Variations of this approach analyze the glycopeptides by MALDI-TOF MS following their isolation by either reversed-phase highperformance liquid chromatography (HPLC) or some means of glycan-specific capturing [6]. This step, however, potentially deprives the analyst of information about the extent of glycan truncation or incomplete site occupancy.

An extreme variety of the "glycopeptide" approach uses ESI-MS of intact antibodies [9, 10, 12]. ESI-MS analysis of individual heavy and light chains obtained by reduction is less in danger of occluding subtle mass differences. This may be less of a concern, however, given the enormous resolution powers of the latest generation mass spectrometers of the Q-TOF and orbitrap type (and certainly of the ion-cylcotron type). Intact antibody analysis gives information on the combination of glycoforms in the assembled antibody. Information on glycan pairing has also been obtained from papain-derived IgG-Fc preparations [13, 14].

In regard to the glycan strategy, it is noteworthy to point out that all $\mathrm{N}$-glycans of the sample, including those in the $\mathrm{Fc}$ and the Fab fragments and those from impurities, are released and analyzed together.

The methods dealing with free glycans again fall into two categories: chromatographic and mass spectrometric methods, which come together in their Hegelian synthesis, LC-MS $[6,15]$. HPLC is usually performed with fluorescently labeled glycans, with 2-aminobenzoic acid and its amide being the most widely used reagents [16]. One reason for this preference over the older 2-aminopyridine is the milder reaction conditions. However, conditions for 2aminopyridine can be chosen that bring about a similarly low degree of desialylation as 2-aminobenzamide (Table I). In any case, $\alpha 2,3$-linkages are more prone to hydrolysis than $\alpha 2,6$-linkages [16].

Table I Sialic acid loss by reductive amination as determined by normal-phase HPLC

\begin{tabular}{llll}
\hline & $\alpha 2,6(\%)$ & $\alpha 2,3(\%)$ & Yield (\%) \\
\hline AB 1 & 1.7 & 4.9 & 90 \\
AB 2 & 0.5 & 1.2 & 60 \\
PA 1 & 13.6 & 81.9 & 95 \\
PA 2 & 2 & 5.2 & 90 \\
\hline
\end{tabular}

Derivatization of pure disialylated $N$-glycans (either $\mathrm{Na}^{6-4} \mathrm{Na}^{6-4}$ or $\mathrm{Na}^{3-4} \mathrm{Na}^{3-4}$ ) with 2-aminobenzamide was performed by the classical method [16] for 2 hours at $70^{\circ} \mathrm{C}(\mathrm{AB} 1)$ or overnight at $37^{\circ} \mathrm{C}(\mathrm{AB} 2)$. Derivatization with 2-aminopyridine was done by the original overnight method (PA 1) or by the modified mild procedure (PA 2) [16]. Derivatization yield was determined by ESI-MS.

\section{Concomitant Analysis of Neutral and Sialylated $N$-Glycans}

Underivatized glycans can be analyzed by mass spectrometry, which, however, is inherently problematic when analytes of different dissolved-state charge are compared. In MALDI-MS, neutral and sialylated glycans can be analyzed separately using different matrices and ion polarity, or turned into neutral species by permethylation [6]. In ESI-MS, all types of sugars can be observed in both ion modes, however, with different detection sensitivity (see next section).

A recent interlaboratory study insinuated that comparative quantitation of neutral and sialylated $N$-glycans (from antibodies) is a task that is still beyond current technical abilities [17]. The study compared the ratios of neutral, monosialylated, and disialylated glycans of $\mathrm{IgG}$ as obtained from 10 laboratories using various methods. Values from about $6 \%$ to $26 \%$ for monosialylated glycans and $1 \%$ to $13 \%$ for disialylated glycans in human IgG were reported [17]. The study revealed a glaring difference between chromatographic and mass spectrometric methods, a deviation that was neither found by another large study [11], nor by the authors of this review.

The fluorescence yield of glycans labeled (e.g., with 2aminobenzamide) can be assumed to be independent of terminal oligosaccharide modifications. Thus, peak areas are understood as faithfully reflecting the amount of a given oligosaccharide, although the peak area may to some extent be influenced by the solvent composition changing during chromatography, e.g., by normal-phase HPLC on amidesilica [18] and by insufficiently separated components. More reliable results may be obtained with blends of pure compounds than with natural glycan mixtures.

A comparison of the ratios obtained by normal-phase HPLC and ESI-MS of glycopeptides revealed a good match between the two methods [7]. This indicated that both results essentially reflected the real composition. This was not at all the case when free glycans were subjected to LCESI-MS in negative mode, where disialylated sugars gave disproportionately large peaks. In contrast, positive mode detection yielded peak ratios similar to those obtained by fluorescence HPLC [19].

\section{Glycosylation of IgG-Update}

$\mathrm{N}$-glycans on IgG are variations on the theme of "diantennary glycan with fucose, sialic acid, and bisecting GlcNAc." Chromatographic analyses by reversed- and normal-phase HPLC gave a valuable synopsis including resolution of isomers [18, 20, 21]. The data from this pioneering period are still valid. Minor corrections were 
brought about by LC-ESI-MS work with regard to the abundance of disialylated structures in the $\mathrm{Fc}$ region $[8,22]$, which tended to be overestimated in earlier studies. This was probably due to contamination of $\mathrm{Fc}$ preparations with Fab fragments. (A comprehensive table of the glycans that occur on $\operatorname{IgG}$ and $\mathrm{Fc}$ and $\mathrm{Fab}$ fragments with a "Rosetta stone" of the relevant structure names and schemes can be found at http://www.proglycan.com.)

Monogalactosylated glycans from IgG show a preponderance of the isomer with galactose on the 6-arm [18, 20], which arises from the special steric situation of the $\mathrm{Fc}$ glycans in IgG1. Remarkably, in $\operatorname{IgG} 2$, the $\mathrm{AGnF}$ and GnAF isomers occur in even amounts as found on myeloma antibodies [18, 20] as well as on isolated $\operatorname{IgG} 2$ glycopeptides from polyclonal $\operatorname{IgG}$ (data not shown). Enzyme specificity is the reason for the strong-arm preference of IgG sialylation [18, 20], which was found to occur on both Fab and Fe glycans [22]. This topology of monosialylated $N$-glycans was recently corroborated by a nuclear magnetic resonance study [23]. Bisected glycans are more prominent in the Fab region, where even the platonic ideal of a disialylated bisected and fucosylated structure can be found.

The amount of variable region glycosylation was estimated at $20 \%$ from sequence database entries [24] and reported to be $15 \%$ to $20 \%$ [20] or $25 \%$ [25], but experimental evidence for these figures is not readily accessible. We thus attempted a recalculation of the degree of Fab glycosylation. Among Fab glycans, $46.2 \%$ are sialylated [22]. This Fab fraction constitutes $12.3 \%$ of all Fab fragments as revealed by chromatography on a sialic acid-binding lectin. This translates into $26.7 \%$ of $\mathrm{Fab}$ fragments being glycosylated with an error estimate of $\pm 2.5 \%$, which should also cover errors due to a possible small amount of diglycosylated Fabs.

\section{Lectin Fractionation of IVIG}

Kaneko and colleagues [4] found that the Sambucus nigra agglutinin (SNA)-binding fraction of IVIG had a roughly 10-fold higher anti-inflammatory potency compared with total IVIG. The relative potency of the nonbinding fraction has not been reported. However, as $12 \%$ of IVIG bind to SNA [22], one might speculate that this lectin-affinity fraction holds the entire anti-inflammatory activity, with the nonbinding fraction being essentially ineffective. At first glance, these results appear to match nicely with the $11 \%$ of sialylated $N$-glycans found in the $\mathrm{Fc}$ region $[8,22]$. The situation, however, is more complex.

Site-specific analysis of the SNA binding and nonbinding fractions of IVIG revealed no significant difference in Fc sialylation [22]. The obvious conclusion was that the fractionation of $\operatorname{IgG}$ on SNA was solely based on the $\mathrm{N}$ - glycans in the variable domains, whereas the sialoglycans in the $\mathrm{C}_{\mathrm{H}} 2$ domain were inaccessible to the lectin. This view was seemingly supported by SNA fractionation of isolated $\mathrm{Fab}$ fragments [22]. However, it harshly contradicts the earlier conclusion that the anti-inflammatory activity depended on sialylation of the Fc region $\mathrm{N}$-glycans [3, 4]. Stadlmann and coworkers might have put that contradiction on their banner, were it not for the mere coincidence that they came to analyze the mAb $4 \mathrm{E} 10$, produced in Nawalma cells, which had an unusually high degree of monosialylated $\mathrm{N}$-glycans. These glycans are $\alpha 2,6$-sialylated and confined to the $\mathrm{Fc}$ region. If only $\mathrm{Fab}$ sialylation would confer SNA binding, the 4E10-hum should not bind to this lectin. Unexpectedly, a large fraction of the mAb did bind and large amounts of monosialylated $\mathrm{N}$-glycans were found in both the binding and nonbinding fraction. This phenomenon could be explained by looking at the whole antibody with its $2 \mathrm{Fc}$ glycans. The stochastic prediction of the percentage of mAbs with 0,1 , and 2 sialic acids matched the experimentally determined value of SNA binding of $43 \%$ only under the premise that two sialic acids were required for SNA binding. Such glycoforms could either contain disialylated and nonsialylated glycans (S2-S0 glycoform) or two monosialylated $N$-glycans (S1-S1 glycoform) (Fig. 1). These considerations assume that all combinations of glycans occur with equal probability. At least up to the $\mathrm{S} 1-\mathrm{S} 0$ combination, this assumption is substantiated by ESI-MS analysis of $\mathrm{Fc}$ fragments or whole antibody [13].

Addressing IVIG with this finding in mind, we realized that $11 \%$ of $\mathrm{S} 1$ glycans could result in about $1 \%$ of the $\mathrm{S} 1-$ S1 glycoform (Table II). Indeed, this correlates with the result of fractionation of Fc on SNA [22]. Notably, this requirement for two sialic acids for SNA binding does not extend to other glycoproteins, as Fab fragments with one sialic acid did bind (data not shown). We assume that the polypeptide strands of the $\mathrm{C}_{\mathrm{H}} 2$ domain shield one sialic acid residue. Denaturation should obstruct this shielding, and indeed, heat treatment of the SNA-nonbinding IVIG fraction led to strong SNA binding (Alfred Weber, personal communication, 2009).

Kaneko and colleagues [4] found a significant increase in anti-inflammatory efficacy for the SNA-retained fraction of IVIG. As we have shown that the percentage of total sialylated glycans in the Fc region is hardly altered by SNA fractionation, this means that glycoforms with just one sialic acid occur in both pools and must therefore be regarded as more or less ineffective. From that, it was argued that the double sialylation of IgG constitutes the minimal requirement for both the binding to SNA and its anti-inflammatory effect [22]. Hypothetically, the antiinflammatory power could also reside in glycoforms with three or four sialic acids, for whose actual existence there is currently no experimental evidence and would in theory 
Fig. 1 Sialylation of the Fc fragment. The Fc fragment of an antibody (taken with Pymol from 1HZH.PDB; top picture). The $\mathrm{C}_{\mathrm{H}} 2$ domain in all conceivable glycoforms in frontal view and-except for the hypersialylated glycoforms - in side view (bottom picture). In the last row, only a second sialic acid (purple diamond) is exposed and accessible to a lectin

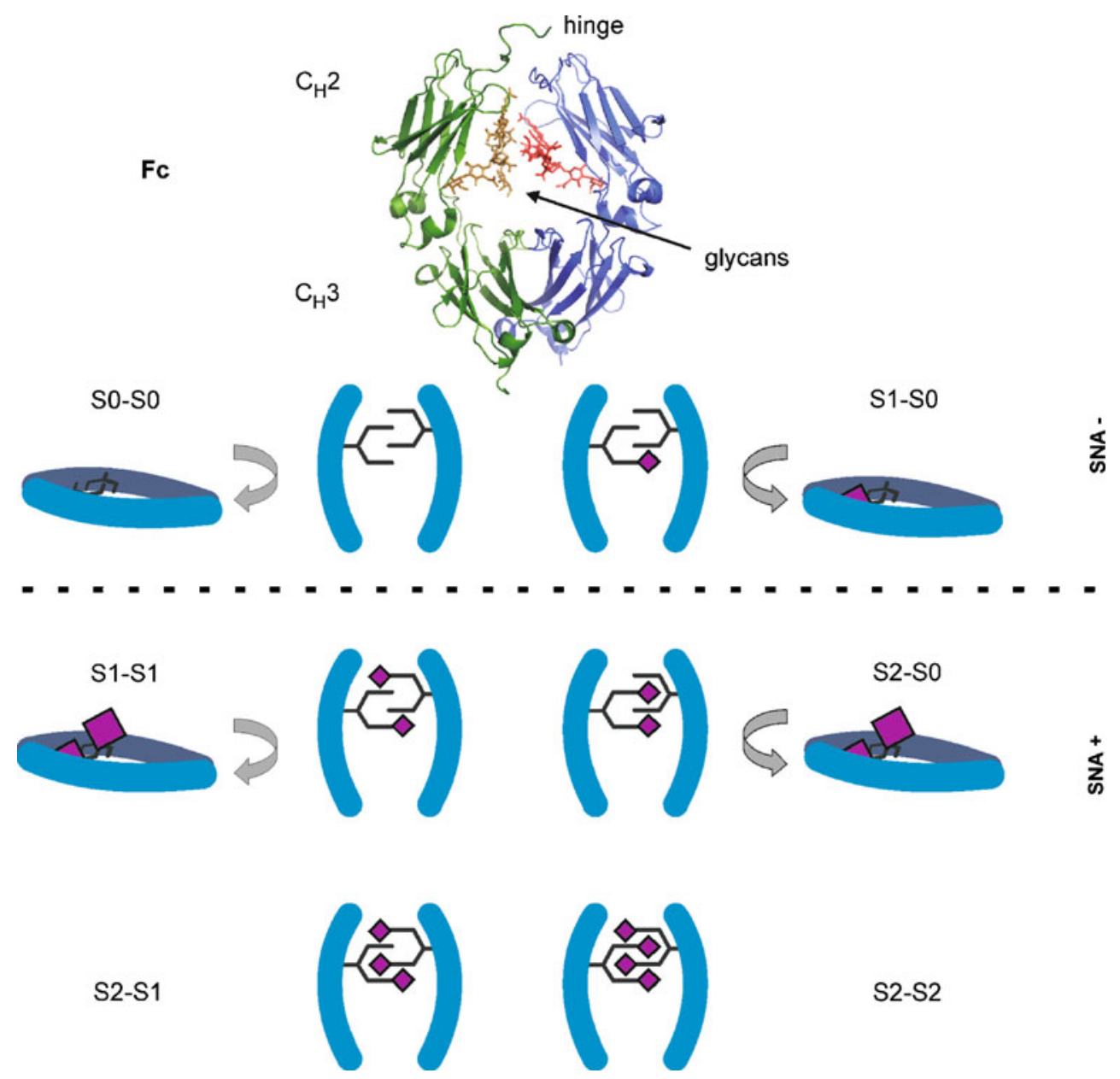

account for less than $0.02 \%$ of all human IgG glycoforms (Table II). As it is very difficult to add sialic acid to IgG beyond the S1-S1 glycoform [23], it appears important to clarify whether such hypersialylation would increase the

Table II Calculated abundance of sialylated glycoforms of IgG. The table on the left shows the combinations of glycans in the anti-HIV $\mathrm{mAb}$ 4E10-hum [7]; the table on the right shows combinations of anti-inflammatory potency. Such experiments could be done with mAbs, where a separation according to the number of sialic acids is possible. Considering the spatial constraints in the Fc glycosylation pocket [2], a most

glycans for human polyclonal IgG. $p$ is the probability factor derived from the percentage of glycans with 0,1 , or 2 sialic acids irrespective of other structural features

\begin{tabular}{|c|c|c|c|c|c|c|c|c|c|}
\hline \multicolumn{5}{|c|}{ mAb 4E10-hum } & \multicolumn{5}{|l|}{ IVIG } \\
\hline \multirow[t]{2}{*}{ Sia \# } & & So & $\mathrm{S} 1$ & $\mathrm{~S} 2$ & \multirow[t]{2}{*}{ Sia \# } & & S0 & $\mathrm{S} 1$ & $\mathrm{~S} 2$ \\
\hline & $p$ & 0.398 & 0.541 & 0.061 & & $p$ & 0.896 & 0.104 & 0.0005 \\
\hline So & 0.398 & 0.158 & 0.215 & 0.024 & So & 0.896 & 0.8028 & 0.0932 & 0.0004 \\
\hline S1 & 0.541 & 0.215 & 0.293 & 0.033 & S1 & 0.104 & 0.0932 & 0.0108 & 0.0001 \\
\hline S2 & 0.061 & 0.024 & 0.033 & 0.004 & $\mathrm{~S} 2$ & 0.0005 & 0.0004 & 0.0001 & 0.0000 \\
\hline \multirow{2}{*}{\multicolumn{3}{|c|}{ SNA nonbinding (\%) }} & \multicolumn{2}{|c|}{ SNA binding (\%) } & \multirow{2}{*}{\multicolumn{2}{|c|}{ SNA nonbinding (\%) }} & & \multicolumn{2}{|c|}{ SNA binding (\%) } \\
\hline & & & $\mathrm{S} 1-\mathrm{S} 1$ & 29.3 & & & & S1-S1 & 1.08 \\
\hline S0-S0 & 15.8 & & $\mathrm{~S} 2-\mathrm{S} 0$ & 4.9 & S0-S0 & 80.3 & & $\mathrm{~S} 2-\mathrm{S} 0$ & 0.09 \\
\hline \multirow[t]{2}{*}{$\mathrm{S} 1-\mathrm{S} 0$} & 43.1 & & $\mathrm{~S} 2-\mathrm{S} 1$ & 6.6 & S1-S0 & 18.6 & & $\mathrm{~S} 2-\mathrm{S} 1$ & 0.01 \\
\hline & & & $\mathrm{S} 2-\mathrm{S} 2$ & 3.3 & & & & $\mathrm{~S} 2-\mathrm{S} 2$ & 0.01 \\
\hline Sum & 58.9 & & Sum & 44.0 & Sum & 98.9 & & Sum & 1.2 \\
\hline
\end{tabular}

mAb indicates monoclonal antibody; IVIG, intravenous IgG; SIA, sialic acids; SNA, S. nigra agglutinin. 
interesting question to raise is "what is the impact of a high degree of sialylation of Fc glycans on protein structure?"

Acknowledgments We thank Dr. Alfred Weber (Baxter BioScience, Vienna) for his valuable suggestions and Dr. Florian Rüker (BOKU, Vienna) for help with the protein structure illustrations and Dr. Mark Schiefermeier (Sandoz $\mathrm{GmbH}$ ) for discussion on AB labeling.

Open Access This article is distributed under the terms of the Creative Commons Attribution Noncommercial License which permits any noncommercial use, distribution, and reproduction in any medium, provided the original author(s) and source are credited.

\section{References}

1. Jefferis R. Glycosylation as a strategy to improve antibody-based therapeutics. Nat Rev Drug Discov. 2009;8:226-34.

2. Raju TS. Terminal sugars of $\mathrm{Fc}$ glycans influence antibody effector functions of IgGs. Curr Opin Immunol. 2008;20:471-8.

3. Anthony RM, Nimmerjahn F, Ashline DJ, Reinhold VN, Paulson JC, Ravetch JV. Recapitulation of IVIG anti-inflammatory activity with a recombinant IgG Fc. Science. 2008;320:373-6.

4. Kaneko Y, Nimmerjahn F, Ravetch JV. Anti-inflammatory activity of immunoglobulin $\mathrm{G}$ resulting from $\mathrm{Fc}$ sialylation. Science. 2006;313:670-3.

5. Wuhrer M, Porcelijn L, Kapur R, Koeleman CA, Deelder A, de Haas $\mathrm{M}$, et al. Regulated glycosylation patterns of $\operatorname{IgG}$ during alloimmune responses against human platelet antigens. J Proteome Res. 2009;8:450-6.

6. Huhn C, Selman MH, Ruhaak LR, Deelder AM, Wuhrer M. IgG glycosylation analysis. Proteomics. 2009;9:882-913.

7. Stadlmann J, Pabst M, Kolarich D, Kunert R, Altmann F. Analysis of immunoglobulin glycosylation by LC-ESI-MS of glycopeptides and oligosaccharides. Proteomics. 2008;8:2571-858.

8. Wuhrer M, Stam JC, van de Geijn FE, Koeleman CA, Verrips CT, Dolhain RJ, et al. Glycosylation profiling of immunoglobulin $\mathrm{G}$ (IgG) subclasses from human serum. Proteomics. 2007;7:4070-81.

9. Beck A, Bussat MC, Zorn N, Robillard V, Klinguer-Hamour C, Chenu $\mathrm{S}$, et al. Characterization by liquid chromatography combined with mass spectrometry of monoclonal anti-IGF-1 receptor antibodies produced in $\mathrm{CHO}$ and NS0 cells. J Chromatogr B Analyt Technol Biomed Life Sci. 2005;819:203-18.

10. Sinha S, Pipes G, Topp EM, Bondarenko PV, Treuheit MJ, Gadgil HS. Comparison of LC and LC/MS methods for quantifying $N$ glycosylation in recombinant IgGs. J Am Soc Mass Spectrom. 2008;19:1643-54.

11. Wada Y, Azadi P, Costello CE, Dell A, Dwek RA, Geyer H, et al. Comparison of the methods for profiling glycoprotein glycans-
HUPO Human Disease Glycomics/Proteome Initiative multiinstitutional study. Glycobiology. 2007;17:411-22.

12. Olivova P, Chen W, Chakraborty AB, Gebler JC. Determination of $N$ glycosylation sites and site heterogeneity in a monoclonal antibody by electrospray quadrupole ion-mobility time-of-flight mass spectrometry. Rapid Commun Mass Spectrom. 2008;22:29-40.

13. Mimura Y, Ashton PR, Takahashi N, Harvey DJ, Jefferis R Contrasting glycosylation profiles between $\mathrm{Fab}$ and $\mathrm{Fc}$ of a human IgG protein studied by electrospray ionization mass spectrometry. J Immunol Methods. 2007;326:116-26.

14. Mimura Y, Ghirlando R, Sondermann P, Lund J, Jefferis R. The molecular specificity of $\mathrm{IgG}-\mathrm{Fc}$ interactions with $\mathrm{Fc}$ gamma receptors. Adv Exp Med Biol. 2001;495:49-53.

15. Pabst M, Bondili JS, Stadlmann J, Mach L, Altmann F. Mass+ retention time $=$ structure: a strategy for the analysis of $\mathrm{N}$-glycans by carbon LC-ESI-MS and its application to fibrin $\mathrm{N}$-glycans. Anal Chem. 2007;79:5051-7.

16. Pabst M, Kolarich D, Pöltl G, Dalik T, Lubec G, Hofinger A, et al. Comparison of fluorescent labels for oligosaccharides and introduction of a new postlabeling purification method. Anal Biochem. 2009;384:263-73.

17. Thobhani S, Yuen CT, Bailey MJ, Jones C. Identification and quantification of $N$-linked oligosaccharides released from glycoproteins: an inter-laboratory study. Glycobiology. 2009;19:201-11.

18. Guile GR, Rudd PM, Wing DR, Prime SB, Dwek RA. A rapid high-resolution high-performance liquid chromatographic method for separating glycan mixtures and analyzing oligosaccharide profiles. Anal Biochem. 1996;240:210-26.

19. Pabst M, Altmann F. Influence of electrosorption, solvent, temperature, and ion polarity on the performance of LC-ESI-MS using graphitic carbon for acidic oligosaccharides. Anal Chem. 2008;80:7534-42.

20. Holland M, Yagi H, Takahashi N, Kato K, Savage CO, Goodall $\mathrm{DM}$, et al. Differential glycosylation of polyclonal $\mathrm{IgG}$. IgG-Fc and $\mathrm{IgG}-\mathrm{Fab}$ isolated from the sera of patients with ANCAassociated systemic vasculitis Biochim Biophys Acta. 2006;1760:669-77.

21. Jefferis R, Lund J, Mizutani H, Nakagawa H, Kawazoe Y, Arata $\mathrm{Y}$, et al. A comparative study of the N-linked oligosaccharide structures of human IgG subclass proteins. Biochem J. 1990;268:529-37.

22. Stadlmann J, Weber A, Pabst M, Anderle H, Kunert R, Ehrlich HJ, et al. A close look at human IgG sialylation and subclass distribution after lectin fractionation. Proteomics. 2009;9:4143-53.

23. Barb AW, Brady EK, Prestegard JH. Branch-specific sialylation of IgG-Fc glycans by ST6Gal-I. Biochemistry. 2009;48:9705-7.

24. Jefferis R. Antibody therapeutics: isotype and glycoform selection. Expert Opin Biol Ther. 2007;7:1401-13.

25. Arnold JN, Wormald MR, Sim RB, Rudd PM, Dwek RA. The impact of glycosylation on the biological function and structure of human immunoglobulins. Annu Rev Immunol. 2007;25:21-50. 